\title{
Understanding the Ageing Process, Recovering Phase and Fault Diagnosis of Electrochemical Double Layer Capacitors
}

\author{
Dr. Dimitri Torregrossa ${ }^{1}$, Dr. Kathryn E. Toghill ${ }^{2}$, Pr. Hubert H. Girault ${ }^{2}$, Pr. Mario Paolone ${ }^{1}$
}

1: Distributed Electrical System Laboratory at Swiss Federal Institute of Technology in Lausanne, Switzerland.

2: Laboratory of Physical and Analytical Electrochemistry, Ecole Polytechnique Fédéral de Lausanne, Switzerland.

\begin{abstract}
This paper focuses on the description of the behavior of supercapacitors during over-temperature and overvoltage stresses and the consequent ageing processes. Specific physical evidences supporting the explanation of the experimental findings are provided and discussed in the paper. A method to distinguish between over-temperature and overvoltage post-faults of these storage devices is summarized and discussed. Additionally, new accelerated ageing phases and recovering phases are detected during combined high-pulsed Power Cycling and high-temperature Life endurance tests.
\end{abstract}

Keywords- Supercapacitors, fault diagnosis, ageing, recovering phase, accelerated ageing.

\section{INTRODUCTION}

As known, supercapacitors (SCs) are electrical storage devices used in several applications requiring high power densities. The power that can be extracted from a SC depends on its size, state-of-charge $(\mathrm{SoC})$ and on its state-of-health $(\mathrm{SoH})$. This last quantity is extremely important since it directly depends on the equivalent series resistance $(E S R)$ of the SC and on its capacitance $C_{S C}$ : two parameters that can be easily evaluated.

The literature concerning the SC SoH, and relevant fault diagnosis, is quite limited and can be summarized by the works [1]-[4]. As discussed in these papers, SC failures appear during ageing causing degradation of their energetic and power performances. In several applications the improvement of SC reliability is fundamental and evaluation of the $\mathrm{SoH}$ remains a priority in order to ensure timely maintenance interventions.

In [1] the SC diagnosis, during operating condition characterized by the SC temperature $\left(T_{S C}\right)$ and SC voltage $\left(V_{S C}\right)$ not exceeding the nominal values, has been inferred by comparing the $V_{S C}$ slope in charging/discharging with the corresponding values estimated prior to ageing. The Authors of [3] and [4] focused on the observation of the changes in the electrochemical properties of generic organic electrolytes (also used in SC) when they are stressed with high temperatures and voltages. None of the above studied the performance losses of SC when they are stressed with $T_{S C}$ and $V_{S C}$ above the nominal values (for $T_{S C}$ we refer to the electrolyte boiling point generally in the order of $75^{\circ} \mathrm{C}$, and for $V_{S C}$ to the decomposition voltage generally in the order of $3 \mathrm{~V}$ ).

As expected, an accurate SC $\mathrm{SoH}$ estimation is based on the exact knowledge of the $\mathrm{SC}$ ageing processes. A thorough study of the literature related to the experimental investigation of the SC ageing mechanism has been presented in [5]-[9]. In these studies two main types of tests have been performed in order to detail the ageing mechanism: i) Life Endurance (LE) and ii) Power Cycling (PC) tests. The LE test allows for evaluating the effects of $V_{S C}$ and $T_{S C}$ on the SC life; PC tests analyze the effects of the SC extracted current on its lifetime. Combined PC and LE tests take into account all the possible ageing factors of SCs. Notably, some Authors (i.e., [5] and [6]) have also identified a particular stage, called the $\mathrm{SC}$ recovering phase, taking place once the PC is stopped. This peculiar phase is characterized by a temporary increase of the SC capacitance $C_{S C}$. The consequence is a change in the $\mathrm{SoH}$ and in the power that can be extracted from the SC. However, in the above-listed references, the physical reasons for the recovering phase have not been accurately analyzed and identified. In particular, the effects of $T_{S C}$ on the duration and on the entity of this recovery phase, along with the conditions to be verified to enable this phenomenon, have not been fully investigated.

This work aims at contributing the understanding of these ageing phenomena and the peculiarity of the recovering phase. Furthermore, new sub-phases named accelerated ageing phases, during which higher $C_{S C}$ deterioration has been observed, have been observed and studied for the first time.

Another contribution of the paper concerns the fault diagnosis of SCs. A SC can be non-intentionally stressed with high $T_{S C}$ and $V_{S C}$ exceeding the nominal values. The dynamic behavior of the $\mathrm{SC}$, together with the physical damage incurred after having achieved these extreme operating conditions, have been investigated and analyzed. In particular, the paper proposes a method to distinguish between SC faults associated to over-temperatures $v$ s over-voltages based on the ESR and the $V_{S C}$ decay observed during the SC charge-redistribution.

\section{SCS FAULT DIAGNOSIS FOR HIGH TEMPERATURE AND HIGH VOLTAGE STRESSES}

\section{A. Analysis of SCs behaviour after high-temperature stresses}

In this section, as well as in the rest of the paper, we make reference to a group of electric double layer capacitors (EDLC) having a nominal capacitance of $365 \mathrm{~F}$ provided by the same manufacturer.

SC manufactures generally declare a maximum storage temperature $T_{M A X}$ of $70^{\circ} \mathrm{C}$ and a maximum operating temperature of $65^{\circ} \mathrm{C}$. The information related to the performances and the dynamic behavior of SC when the $T_{S C}$ passes the above limits during cycling have not yet been investigated and neither declared by SC manufactures. In this respect, seven over-temperature tests have been performed respectively at $75^{\circ} \mathrm{C}, 95^{\circ} \mathrm{C}, 115^{\circ} \mathrm{C}, 130^{\circ} \mathrm{C}$ on the same $\mathrm{SC}$ in order to investigate its performance changes compared to those 
at $25^{\circ} \mathrm{C}$. Additionally, once the $\mathrm{T}_{S C}=130^{\circ} \mathrm{C}$ was reached, it was held at that temperature for 72 hours and the SC performances have been evaluated every 24 hours. The capacitor was charged to $2.35 \mathrm{~V}$ in each experiment.

As already discussed in [5]-[9], temperature is the main source of SC ageing since it directly acts on the micropore structure of the electrodes. The changes provided by over temperature stresses are not reversible and, at nominal temperature values (i.e. below $T_{M A X}$ ), the main effect is a decrease of $C_{S C}$. However, temperature values above the recommended ones lead to an important structural modification of the SC electrodes combined with changes of the electrolyte physical properties (i.e., conductivity, viscosity and aggregation properties [3]).

The observed effects (summarized in Table I., Fig. 1 and Fig. 2) are: i) an expected increase of the ESR (the value can double after 72 hours of over temperature stress), ii) an unexpected increase of the SC voltage decay during short relaxation phases ( $V_{20}$ is the value of $V_{S C}$ measured 20 minutes after the power generator is turned off), iii) an unexpected $C_{S C}$ increase and a corresponding increase of the SC charging time.

Concerning the fast increase of the ESR, it is due to the fact that the normal process leading a LE test is extremely accelerated. Indeed, the ESR is composed by two main contributions: the resistance related to the electrode-electrolyte surface interface $\left(S_{S C}\right)$ and the resistance related to the electrolyte itself. The extreme high temperature modifies the chemical properties of the electrolyte and it produces overpressures in the restricted SC volume causing size modification of the electrode's micropore structure. Consequently, the $S_{S C}$ is modified and, in general, decreased. Concerning the SC decay voltage, an increase of the SC selfdischarge resistance displayed as a more pronounced voltage decay, was expected and observed. It is well-known that the SC self-discharge process requires long observation times, thousands of seconds after the SC open circuit voltage $\left(V_{O C}\right)$ has been reached. Fig. 1 shows the $V_{S C}$ profiles during charging and redistribution phases at various temperatures. Notably, the decay voltage observed in Fig. 1 is not related to the selfdischarge originated by a charge-leakage phenomenon but is related to changes in the redistribution of the residual charge phenomenon (see [6] for further details). Indeed, at higher temperatures the redistribution phenomena of the charges stored inside the SC is less important during both the charging and the relaxation phases. This involves a lower value of SC residual charges $(\Delta Q)$ stored inside the SC [6]. Concerning the $C_{S C}$ increase, at higher temperatures the electrolyte has a higher ionic conductivity (ion mobility) [10] and, consequently, the charge extracted by the SC increases: this results in a capacitance increase. The $C_{S C}$ increase, along with a mitigated redistribution phenomenon at higher temperature, justifies the increase of the charging time. All these observations suggest a possible fault diagnosis detection after over-temperatures in $\mathrm{SC}$ by leveraging on the meaning of the SC decay voltage and capacitance. The entire procedure followed to evaluate the electric parameters of the SC is described in [5].

In order to investigate the capability of the SC to deliver pulsed currents accounting also the over-temperature stresses, Fig. 2 compares the current delivered by the SC at different temperatures. The profile of the extracted current has been a- priori defined. This profile is characterized by a fast dynamic with a peak current value 5 times higher than the SC nominal one. As can be observed, the reduction of the peak current for $T_{S C}$ below $95^{\circ} \mathrm{C}$ is negligible; after 72 hours at $130^{\circ} \mathrm{C}$ the current decreases by $4 \%$. In view of these results, it is possible to conclude that the SC was still able to provide highly pulsed currents in spite of over-temperature stresses.

\section{B. Analysis of SC behaviour after high-voltage stresses}

The manufactures of SC generally declare a maximum voltage $V_{M A X}$ to be applied to the SC. This value is equal, in general, to $2.85 \mathrm{~V}$. To the best of the authors knowledge, the performances and dynamic behavior of SCs when the $V_{S C}$ passes the above limit have not been comprehensively investigated in the existing literature.

In this respect, we have conducted an experimental investigation where five over-voltage tests have been performed at $3 \mathrm{~V}, 3.25 \mathrm{~V}, 3.5 \mathrm{~V}, 3.75 \mathrm{~V}$ and $4 \mathrm{~V}$ on the same $\mathrm{SC}$. The performance changes have been compared to those ones when it is charged at $2.45 \mathrm{~V}$. The temperature was maintained at $25^{\circ} \mathrm{C}$.

The observed results (summarized on Table II, Fig. 3 and Fig. 4) are: i) an unexpected large increase of the ESR (this parameter can be 10 times bigger than the value measured at the nominal voltage), ii) an unexpected increase of the decay voltage during short relaxation phases, iii) an expected $C_{S C}$ decrease.

Fig. 3 shows the $V_{S C}$ profiles during charging and redistribution phases subsequent to various overvoltage stresses applied to the SC. As already explained in the previous subsection, the SC decay voltage observed in Fig. 3 is related to changes in the redistribution of the residual charge phenomenon. This phenomenon depends on the $\Delta Q$ stored in the SC. This latest value depends indirectly on the viscosity of the electrolyte and ion mobility. The main effect of the overvoltage is to change the electrolyte composition [3] and, consequently, it is reasonable to assume that some properties as the viscosity and ion mobility of the electrolyte are subject to changes; consequently the charge penetration into the electrode pores is reduced. This involves a reduction of the $\Delta Q$ along with an important mitigation of the charge redistribution justifying the behavior observed in Fig. 3. Mitigated electrolyte penetration into the electrode pores, along with the reduction of the $S_{S C}$, supports the reduced $C_{S C}$ observed after the overvoltage tests. Similarly to the high-power pulsed tests, performed after the over-temperature stresses, Fig. 4 illustrates the performances of the targeted $\mathrm{SC}$ when it is delivering a current profile with the same one adopted for the overtemperature tests. As can be observed, if the $V_{S C}$ is below 3.50 $\mathrm{V}$, the lost of SC performances are not remarkable. Then, once irreversible modifications of the electrolyte take place, the ESR increases and the $\mathrm{SC}$ is not able to deliver high current values. However, it can still deliver current values close to the nominal one (for the $\mathrm{SC}$ under test this value is equal to $36 \mathrm{~A}$ ). Fig. 5 shows a comparison between SC decay voltages related to the extreme overt-temperature and over-voltage tests, namely $130^{\circ} \mathrm{C}$ after $72 \mathrm{~h}$ and $4 \mathrm{~V}$ during cycling. As it can be observed, the over-voltage effect is more important than the overtemperature one. 


\section{Outlooks}

It is important to underline that the above results have been performed on $4 \mathrm{SCs}$ belonging to the same manufacturer. It is expected to perform a statistical study on a larger set of devices aimed at inferring the distributions of the SC characteristic parameters during these specific operating conditions. It is also expected to perform a SC post-mortem analysis in order to prove the observed degradations.

TABLE I.

SUMMARY OF THE SC CHARACTERISTIC PARAMETERS DURING THE OVER-TEMPERATURE TEST

\begin{tabular}{l|c|c|c}
\hline \multirow{2}{*}{\multicolumn{1}{c}{ Temperature }} & \multicolumn{3}{|c}{$\begin{array}{c}\text { ESR and } \boldsymbol{C}_{\boldsymbol{S C}} \text { evolution during over- } \\
\text { temperature tests }\end{array}$} \\
\cline { 2 - 4 } & $\boldsymbol{E S R}[\mathbf{m} \boldsymbol{\Omega}]$ & $\boldsymbol{C}_{\boldsymbol{S C}}[\mathbf{F}]$ & $\boldsymbol{V}_{\boldsymbol{2 0}}$ \\
\hline $25^{\circ} \mathrm{C}$ & 3.2 & 347 & $2.27 \mathrm{~V}$ \\
\hline $75^{\circ} \mathrm{C}$ & 3.42 & 351 & 2.17 \\
\hline $95^{\circ} \mathrm{C}$ & 3.54 & 357 & 2.13 \\
\hline $115^{\circ} \mathrm{C}$ & 3.7 & 362 & 1.97 \\
\hline $130^{\circ} \mathrm{C}$ & 3.9 & 369 & 1.92 \\
\hline $130^{\circ} \mathrm{C}-24$ hours & 4.9 & 377 & 1.73 \\
\hline $130^{\circ} \mathrm{C}-48$ hours & 5.4 & 412 & 1.76 \\
\hline $130^{\circ} \mathrm{C}-72$ hours & 6.2 & 422 & 1.76 \\
\hline \hline
\end{tabular}

TABLE II.

SUMMARY OF THE SC CHARACTERISTIC PARAMETERS DURING THE OVER-VOLTAGE TESTS

\begin{tabular}{|c|c|c|c|}
\hline \multirow{2}{*}{ Applied Voltage } & \multicolumn{3}{|c|}{$\begin{array}{c}E S R \text { and } C_{S C} \text { evolution during over-voltage } \\
\text { tests }\end{array}$} \\
\hline & $E S R[\mathrm{~m} \Omega]$ & $C_{S C}[\mathbf{F}]$ & $V_{20}$ \\
\hline $2.45 \mathrm{~V}$ & 2.81 & 359.5 & $2.3 \mathrm{~V}$ \\
\hline $3 \mathrm{~V}$ & 2.81 & 370 & $2.3 \mathrm{~V}$ \\
\hline $3.25 \mathrm{~V}$ & 2.81 & 400 & $2.27 \mathrm{~V}$ \\
\hline $3.5 \mathrm{~V}$ & 4.31 & 338 & $2.19 \mathrm{~V}$ \\
\hline $3.75 \mathrm{~V}$ & 6.2 & 296 & $1.87 \mathrm{~V}$ \\
\hline $4 \mathrm{~V}$ & 31 & 285 & $1.62 \mathrm{~V}$ \\
\hline
\end{tabular}

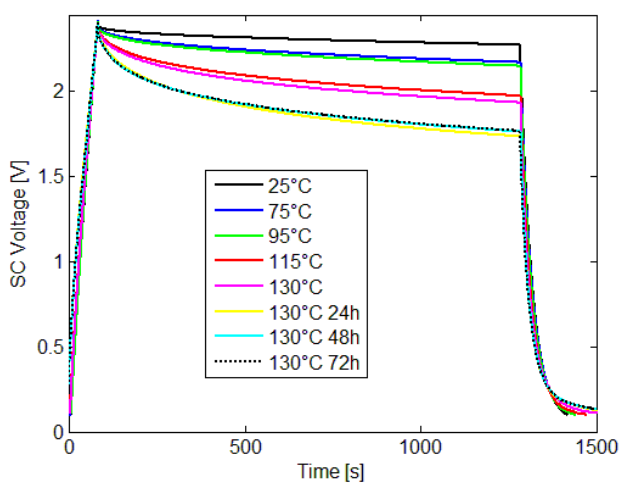

Fig. 1. Time evolution of the SC voltage associated to the charge redistribution at different temperatures.

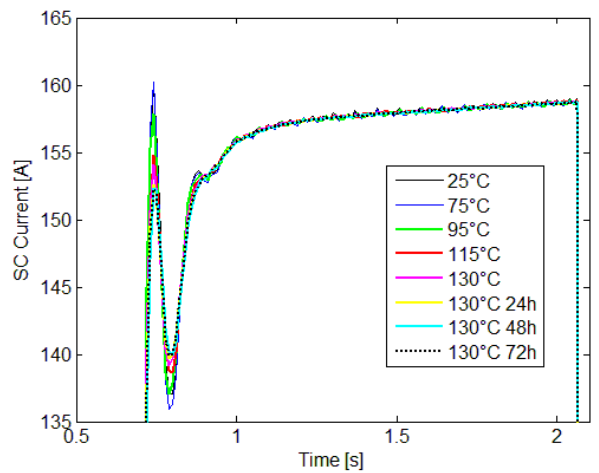

Fig. 2. Profiles of the SC delivered currents during high-power pulsed tests at different temperatures.

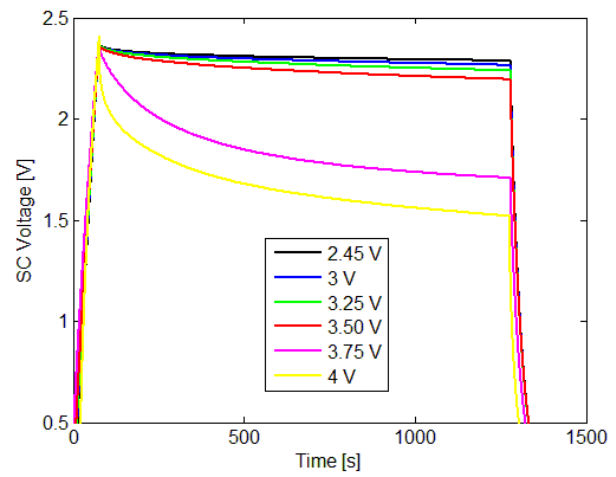

Fig. 3. Time evolution of the SC decay voltage subsequent to over-voltages stresses.

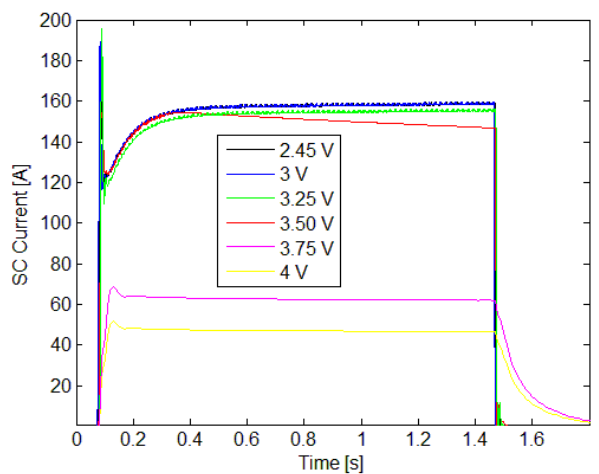

Fig. 4. Profiles of SC delivered current during high-power pulsed tests at different voltages.

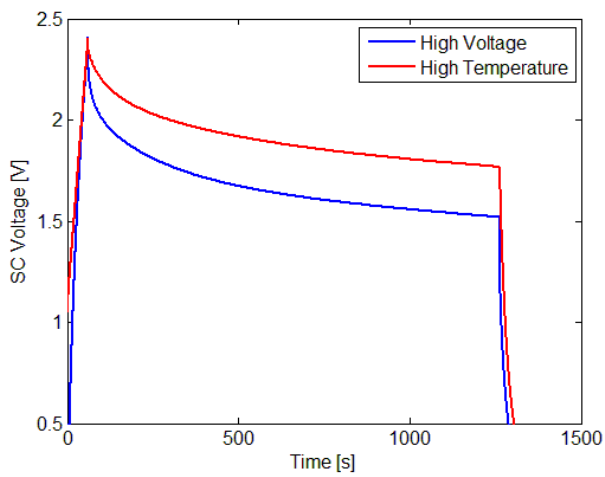

Fig. 5. Time evolution of the SC voltage associated to the charge redistribution for the worst overtemperature and overvoltage tests respectively. 


\section{AGEING AND RECOVERING PROCESS ANALYSIS}

Table III illustrates the evolution of the $C_{S C}$ during a combined LE and PC test at three different temperatures, $25^{\circ} \mathrm{C}$, $35^{\circ} \mathrm{C}$ and $45^{\circ} \mathrm{C}$ respectively (note that a detailed description of the SC ageing tests has been already illustrated in [6]). The structure of the whole ageing test is as follows: the first part is related to the initial combined LE and PC test (time window of 24 hours), a second one related to a single LE test (time window of 12 hours), a third one related to a combined LE and PC test (time window of 136 hours), a fourth one related to a single LE test (time window of 15 hours), a fifth one related to a combined LE and PC test (time window of 106 hours), a sixth one related to a single LE test (time window of 27 hours), a last one related to a combined LE and PC test (time window of 36 hours).

TABLE III.

SUMMARY OF THE $C_{S C}$ EVOLUTION DURING THE COMBINED PC AND LE TESTS

\begin{tabular}{|c|c|c|c|}
\hline \multirow{2}{*}{$\begin{array}{c}\text { Type } \\
\text { of } \\
\text { test }\end{array}$} & \multicolumn{3}{|c|}{$C_{S C}$ evolution [F] } \\
\hline & $T_{S C} 25^{\circ} \mathrm{C}$ & $T_{S C} 35^{\circ} \mathrm{C}$ & $T_{S C} 45^{\circ} \mathrm{C}$ \\
\hline $\mathrm{PC}$ & 354.5 & 353 & 349.5 \\
\hline $\begin{array}{l}21 \\
\text { hours } \\
\text { PC }\end{array}$ & 346.1 (NA) & 345.4 (NA) & 341.5 (NA) \\
\hline LE & 344.6 (AP $-0.45 \%)$ & 344 (AP -0.4\%) & 339.6 (AP $-0.57 \%)$ \\
\hline $\begin{array}{l}9 \\
\text { hours } \\
\text { LE }\end{array}$ & 342.9 (AP $-0.95 \%)$ & 340.9 (AP -1.3\%) & 336.4 (AP -1.5\%) \\
\hline $\mathrm{PC}$ & $346.7(\mathrm{RP}+1.21 \%)$ & $344.2(\mathrm{RP}+0.9 \%)$ & $338.7(\mathrm{RP}+0.7 \%)$ \\
\hline $\begin{array}{l}6 \\
\text { hours } \\
\text { PC }\end{array}$ & $347.3(\mathrm{RP}+1.3 \%)$ & 343.6 (NA +0.7\%) & 338. (NA +0.45\%) \\
\hline $\begin{array}{l}130 \\
\text { hours } \\
\text { PC }\end{array}$ & 340.6 (NA $-0.65 \%$ ) & 338.4 (NA -0.7\%) & 330.4 (NA -1.75\%) \\
\hline LE & 339.4 (AP - $0.3 \%$ ) & 335.8 (AP -0.7\%) & 327.3 (AP -0.95\%) \\
\hline $\begin{array}{l}12 \\
\text { hours } \\
\text { LE }\end{array}$ & 338 (AP $-0.75 \%)$ & 334.7 (AP -1.1\%) & $326.3(\mathrm{AP}-1.2 \%)$ \\
\hline $\mathrm{PC}$ & $339.7(\mathrm{RP}+0.5 \%)$ & $337.7(\mathrm{RP}+0.9 \%)$ & $329.2(\mathrm{RP}+0.9 \%)$ \\
\hline $\begin{array}{l}6 \\
\text { hours } \\
\text { PC }\end{array}$ & $340.7(\mathrm{RP}+0.8 \%)$ & 337.7 (NA +0.9\%) & $329.3(\mathrm{NA}+0.93 \%)$ \\
\hline $\begin{array}{l}100 \\
\text { hours } \\
\text { PC }\end{array}$ & 338.2 (NA $-0.04 \%$ ) & 334.6 (NA - $-0.4 \%$ ) & 327.9 (NA -0.5\%) \\
\hline LE & 336.3 (AP - $0.5 \%)$ & 333.1 (AP -0.4\%) & $325(\mathrm{AP}-0.8 \%)$ \\
\hline 24 & 335.8 (AP $-0.7 \%$ ) & 331 (AP -1\%) & 323.9 (AP -1.2\%) \\
\hline
\end{tabular}

\begin{tabular}{l|c|c|c}
\hline \hline \multirow{2}{*}{$\begin{array}{c}\text { Type } \\
\text { of } \\
\text { test }\end{array}$} & $\boldsymbol{T}_{\boldsymbol{S C}} \mathbf{2 5}^{\circ} \boldsymbol{C}$ & $\boldsymbol{T}_{\boldsymbol{S C}} 35^{\circ} \boldsymbol{C}$ & $\boldsymbol{T}_{S C} 45^{\circ} \boldsymbol{C}$ \\
\cline { 2 - 4 } $\begin{array}{l}\text { hours } \\
\text { LE }\end{array}$ & & & \\
\hline PC & $337.2(\mathrm{RP}+0.4 \%)$ & $333.9(\mathrm{RP}+0.9 \%)$ & $324.2(\mathrm{RP}+0.1 \%)$ \\
\hline $\begin{array}{l}\text { 6 } \\
\text { hours }\end{array}$ & $338(\mathrm{RP}+0.7 \%)$ & $334.2(\mathrm{RP}+0.95 \%)$ & $326.6(\mathrm{RP}+0.8 \%)$ \\
PC & & & \\
\hline $\begin{array}{l}36 \\
\text { hours } \\
\text { PC }\end{array}$ & $337.9(\mathrm{RP}+0.7 \%)$ & $334(\mathrm{NA}+0.9 \%)$ & $325.8(\mathrm{NA}+0.5 \%)$ \\
\hline \hline
\end{tabular}

From Table III two main phases can be identified: (i) accelerated ageing phase and (ii) recovering phase for which the duration and the associated $C_{S C}$ evolution depend strongly on the $T_{S C}$ value.

In Table III the following acronyms are used to clearly identify the different phases: normal ageing phase (NA), accelerated phase (AP) and recovering phase (RP). At each transition the increase or decrease of $C_{S C}$ is evaluated and referred to the last value of the previous ageing phase (LE or combined PC and LE test).

From Table III it is possible to draw the following conclusions: The accelerated ageing phase is detected at any transition of PC test to LE test. The duration seems to be independent from the temperature. However, the associated $C_{S C}$ fade strongly depends on the $T_{S C}$. The recovering phase takes place during the transition of LE to PC test. The condition for which it takes place, along with its duration and entity, depends strongly on the $T_{S C}$ value and on the duration of the previous LE test.

During the LE test and during the combined PC and LE test the $C_{S C}$ has been recorded every 3 hours (note that the procedure that has been used to evaluate the $C_{S C}$ is described in detail in [6]). It is important to note that the observed recovering phase is associated to the utilization of the $\mathrm{SC}$ with high-pulsed current extraction (10 times higher than the nominal value for 1 second). To the best of our knowledge, the available literature does not discuss ageing and recovering processes associated to this particular use of the electrochemical double layer SC.

\section{A. Accelerated ageing phenomenom}

When the SC is aged with a combined PC and LE tests, the $C_{S C}$ decreases followed by a quasi-exponential fading [6]-[9]. The main ageing factor is the current extraction, involving an increase of the $T_{S C}$ and consequent exfoliation of the carbon electrode surface blocking some of its macro-pores. The structure of the electrode consists of elementary crystallites with a large number of interstices between them. The interstices tend to be filled with carbon residues that block the pore entrances during a LE test [10]. This latest effect reduces the $S_{S C}$ and consequently the charge transfer from electrolyte to electrode. It is important to note that some benefits may be associated to higher internal SC temperatures caused by the current extraction: i) a lower electrolyte viscosity increasing the charge penetration of the electrolyte into the pores; ii) higher 
electrolyte conductivity and consequently a higher amount of charge transfer between electrode and electrolyte. Additionally current extraction, especially if the current is pulsed, removes some of the impurities depositing on the above interstices (the quantity of these impurities increases with the temperature). Once the PC is stopped, these above benefits are no longer present, and consequently there is an acceleration of the ageing process, namely a more important and faster $C_{S C}$ decrease. The decrease is more important for the SC that has been previously aged at higher temperature (see in Table III the row associated to the beginning of each LE test). This happens as the higher temperature involves an increase in the production of impurities and further degradation of the electrolyte (discussed below). One may assume that the $I_{S C}$ is a mitigating-ageing factor countering the $T_{S C}$ ageing factor during combined LE and $\mathrm{PC}$ tests.

\section{B. Regenerative phenomenom}

During the LE tests, the $C_{S C}$ follows a quasi-exponential fading $v$ s time that has been already discussed in the literature [6]-[9]. Based on experimental results presented in this paper, the evolution of the $C_{S C}$ during the transition from LE test toward PC test deserves specific analysis. In particular, three different types of $C_{S C}$ behavior, with duration and associated $C_{S C}$ changes that depend strongly on the $T_{S C}$, can be identified at the beginning of the PC restart. The first identified behavior shows an increase in $C_{S C}$ that is maintained for a certain period of time (before returning to age) following the classical capacitance evolution [6]-[9]. The second observed behavior is an increase in $C_{S C}$ for a certain time. After this increase, a normal decrease follows. The third observed behavior is that the $C_{S C}$ increases then suddenly decreases normally.

The occurrence of one of the above behaviors depends on the $T_{S C}$ value and on the duration of the previous LE test. Possible mechanisms describing these behaviors for each $T_{S C}$ are described below.

i) If the $T_{S C}$ is equal to $25^{\circ} \mathrm{C}$ (the nominal temperature specified by the manufacturer) once PC resumes, the first subphase associated to the recovering phenomenon always takes place: the $C_{S C}$ starts to increase until it reaches a maximum value. During this specific phase the extraction of current partially removes some of the impurities that filled the interstices in the previous phase (the LE test). Additionally, the extraction of current involves the increase of temperature with associated decrease of viscosity and increase of the electrolyte conductivity. This results into a larger penetration of the electrolyte into the electrode pores and a higher amount of charge transfer. It is important to note that the current extraction, especially because it has the time evolution characteristic of a pulsed current, is causing micro-cracking in the electrode which modifies the porosity of the electrode [10]. Even if we were not able to have clear evidences, we suppose that the production of these impurities, mixed with the electrolyte, might reduce the electrolyte conductivity as well.

It is reasonable to assume that at the beginning of the PC restart there are two processes: one involving an ageing phenomena associated to current extraction, another one involving a regenerative phenomena associated to the viscosity and electrolyte conductivity changes. During the first part of this phase (6 hours at $25^{\circ} \mathrm{C}$ in Table III), the regenerative phenomena is stronger than the ageing one. Once the $C_{S C}$ reaches the maximum value, it means that the two processes are perfectly compensated. From this moment on, any further extraction of current is no longer compensated by the above benefit causing a reduction of the $C_{S C}$ value. The $C_{S C}$ evolution follows the characteristic fading of a PC test.

At $25^{\circ} \mathrm{C}$, the increase of $C_{S C}$ evaluated at each transition from LE to combined PC and LE test is decreasing: $+1.21 \%$ at the first transition, $+0,5 \%$ and $0.4 \%$ in the last one. It is reasonable to justify this finding by the fact that the benefit associated to the current extraction becomes lower. In fact, the $\mathrm{SC} \mathrm{SoH}$ at the last transition is lower than the one evaluated at the beginning (indeed, the $\mathrm{SoH}$ is mainly related to the filled micropore and the electrolyte properties). At the last transition the benefits associated to the PC are relatively less important than those at the beginning of the test (at the beginning of the test the electrolyte has not deteriorated and few micropores are filled with impurities). The duration of the recovering subphase is still the same at each transition since it is related to an increase in the temperature (increase of electrolyte conductivity and decrease of viscosity) associated to the current extraction. The increase in temperature is practically the same since the heat capacity of the electrolyte is not considerably changed and the current profile used during the PC test is always the same.

ii) If the $T_{S C}$ is equal to $35^{\circ} \mathrm{C}$, once the $\mathrm{PC}$ is restarted, the observation of one of the three above listed possibilities depends on the duration of the previous LE test. As shown in Table III, during the first transition, the recovering phase holds just for 3 hours (the previous LE test held for 12 hours). During the second transition there is a recovering phase that almost compensates the main ageing process (the previous LE test held for 15 hours). During the third transition there is a recovering phase with an associated $C_{S C}$ increase (the previous LE test held for 27 hours).

In order to physically explain the above experimental findings, it is important to underline that during a PC and LE ageing test there is a certain evaporation of the solvent changing the composition of the electrolyte. Some of the evaporated solvent leaks from the $\mathrm{SC}$ which is not totally hermetic, and some of it remains inside the SC creating an overpressure. Based on the consideration reported in [10] it might be possible to assume that the above overpressure modifies the contact between electrode and electrolyte and leads to an additional $C_{S C}$ decrease. This process depends strongly on the $T_{S C}$ value and on the duration of the test. It is reasonable to assume that at the beginning of the whole ageing test and for short durations of LE phases (in our case shorter than 15 hours) the degradation of the electrolyte associated with the LE test is not so important and any benefit related to the extraction of current (during the PC test) is not producing any observable phenomena. After a certain threshold (in our case 15 hours) degradation of the electrolyte takes place and the increase of the temperature and removal of impurities associated to current extraction are temporarily compensating for the whole ageing process. For longer duration LE tests (27 hours) the degradation of the electrolyte, along with the number of micropores filled with impurities, becomes important. Consequently, the extraction of current is associated to important $C_{S C}$ increases. 
iii) If the $T_{S C}$ is equal to $45^{\circ} \mathrm{C}$, once the $\mathrm{PC}$ is restarted, the first sub-phase associated to the recovering phenomena always takes place: the $C_{S C}$ starts to increase until it reaches a maximum value. At the beginning of the test, during the first transition, this phase holds just for 3 hours. For the other transition, since the degradation of the electrolyte is accelerated (indeed, the $T_{S C}$ is higher) the current extraction temporarily benefits the SC. The $C_{S C}$ increase is relatively higher than that evaluated at the same transition at $25^{\circ} \mathrm{C}$. This can be explained with a lower $\mathrm{SC} S \mathrm{SH}$ involving a higher regeneration with current extraction.

\section{Outlooks}

The above results have been inferred on 6 SCs belonging to the same lot provided by the same manufacturer. It is expected to perform a statistical study on a larger set of devices aimed at inferring the distributions of the SC characteristic parameters during these specific transitions. Additionally, the tests were globally performed for 20 days but it would be interesting to predict the behavior of the SCs during the AP and RP over longer test periods. As already stated, the duration and the entity of the above AP and RP depend on solvent evaporation. It would therefore be interesting to provide experimental characterization of the solvent losses during the different ageing tests.

\section{CONCLUSIONS}

This work has described an experimental study aimed at: a) analysing the fault diagnosis of SCs during high temperature and high voltage test; b) discussing the SC state of health and $C_{S C}$ evolution during combined PC and LE tests at different temperatures.

The evolution of the SC characteristic parameters (i.e., ESR and $C_{S C}$ ) under high temperatures (up to $130^{\circ} \mathrm{C}$ ) and high voltages (up to $4 \mathrm{~V}$ ) has been experimentally investigated. Additionally, the ability of the SC to deliver high-pulsed currents, after having achieved the above extreme operating conditions, has been investigated and discussed.

The results show that SCs exhibit a robust behaviour after over-temperature stresses. Conversely, SC behaviour appears quite sensitive to overvoltage stresses. The paper has then proposed a method to infer the type of faults based on the estimation of the SC ESR and on the observation of the SC long-term voltage decay (i.e., the SC voltage during the charge redistribution phase). These observations might represent the basis to develop online and real-time diagnosis tools of SCs.

The results presented in the paper have further allowed for identifying a new recovering phase and an accelerating ageing phase. They appear during combined LE and PC tests at highpulsed currents. In summary, the recovering phase appears at each transition from LE to PC. The duration and behaviour of the $C_{S C}$ increase depends on $T_{S C}$ and on the previous ageing process. The above results have been obtained by investigating the SC behaviour for three different temperatures: the nominal $T_{S C}\left(25^{\circ} \mathrm{C}\right)$, a slightly higher $T_{S C}\left(35^{\circ} \mathrm{C}\right)$ and a moderately high $T_{S C}\left(45^{\circ} \mathrm{C}\right)$.

Additionally, it has observed that the use of high-pulsed currents can be adopted as a method to obtain a temporary increase of the $C_{S C}$.

Based on the above-summarised findings, further research will focus on the investigation of the SC recovering phase including a post mortem analysis to validate the explanations. Further, the results will be used to improve existing analytical $\mathrm{SC}$ ageing models to enable online and real-time $\mathrm{SoH}$ estimators.

\section{ACKNOWLEDGMENT}

The authors gratefully acknowledge the Swiss Innovation and Technology Committee (CTI) to making the experimental characterization of the supercapacitors possible. The Authors thankfully acknowledge M. Robert and M. Burri for their help in carrying out the experimental investigations.

\section{REFERENCES}

[1] A. Oukaoura, B. Tala-Ighila, M. AlSakkab, H. Gualousa, R. Gallayc, B. Boudarta, "Calendar ageing and health diagnosis of supercapacitor", Electric Power System Research 95 (2013), pp. 330-338.

[2] Brian E. Conway, W.G. Pell, T-C. Liu "Diagnostic analyses for mechanisms of self-discharge of electrochemical capacitors and batteries", Journal of Power Sources 65 (1997), pp. 53-59.

[3] A.G. Pandolfo, A.F. Hollenkamp, "Carbon properties and their role in supercapacitors", Journal of Power Sources 157 (2006), pp 11-27.

[4] Tetsuo Nishida, Yasutaka Tashiro, Masashi Yamamoto "Phsycal and electrochemical properties of 1-alkyl-3-methylimidazolium tetrafluoroborate for electrolyte", Journal of Fluorine Chemistry 120 (2003), pp. 135-141.

[5] R. Chaari, O. Briat, J.Y. Deletage, J.Vinassa, "Performances regeneration of supercapacitors during accelerated ageing tests in power cycling," Power Electronics and Applications (EPE 2011), pp.1-7, Aug. 30 2011-Sept. 12011

[6] D. Torregrossa, M. Paolone , "Novel Experimental Investigation of Supercapacitor Ageing during Combined Life-Endurance and PowerCycling Tests," IECON, Vienna, November 2013.

[7] Oliver Bohlen, Julia Kowal, Dirk Uwe Sauer, "Ageing behaviour of electrochemical double layer capacitors Part II. Lifetime simulation model for dynamic applications", Journal of Power Sources 173 (2007) 626-632.

[8] O. Briat , J.-M. Vinassa, N. Bertrand, H. El Brouji, J.-Y. Delétage, E. Woirgard, "Contribution of calendar ageing modes in the performances degradation of supercapacitors during power cycling”, Microelectronics Reliability, vol.50 (2010) 1796-1803.

[9] Wan-Yu Tsaia, Rongying Lina, Shanthi Muralic, Li Li Zhangc, John K. McDonoughd, Rodney S. Ruoffc, Pierre-Louis Tabernaa, Yury Gogotsid, Patrice Simona, "Outstanding performance of activated graphene based supercapacitors in ionic liquid electrolyte from 50 to 80 $1 C$ ", in press in Nano Energy Elsevier.

[10] J. Vetter, P. Novák, M.R. Wagner, C. Veit, K.-C. Möller, J.O. Besenhard, M. Winter, M. Wohlfahrt-Mehrens, C. Vogler, A. Hammouche, "Ageing mechanism in lithium-ion batteries" Journal of Power Sources, Volume 147, Issues 1-2, 9 September 2005, pp. 269281. 